\title{
Running gravitational couplings, decoupling, and curved spacetime renormalization
}

\author{
Antonio Ferreiro $\odot^{*}$ and Jose Navarro-Salas $\odot^{\dagger}$ \\ Departamento de Física Teórica and IFIC, Centro Mixto Universidad de Valencia-CSIC. Facultad de Física, \\ Universidad de Valencia, Burjassot-46100, Valencia, Spain
}

(Received 15 May 2020; accepted 6 August 2020; published 28 August 2020)

\begin{abstract}
We propose to slightly generalize the DeWitt-Schwinger adiabatic renormalization subtractions in curved space to include an arbitrary renormalization mass scale $\mu$. The new predicted running for the gravitational couplings are fully consistent with decoupling of heavy massive fields. This is a somewhat improvement with respect to the more standard treatment of minimal (DeWitt-Schwinger) subtractions via dimensional regularization. We also show how the vacuum metamorphosis model emerges from the running couplings.
\end{abstract}

DOI: 10.1103/PhysRevD.102.045021

\section{INTRODUCTION}

One of the cornerstones in quantum field theory has been the design of regularization/renormalization schemes that allows us to overcome ultraviolet divergences when computing physical observables [1-3]. In perturbative quantum electrodynamics we thus obtain reliable, well-proven results such as the Lamb shift and the running of the electromagnetic coupling constant due to vacuum polarization. The renormalization process always involves an arbitrary mass parameter $\mu$ and the possibility of rescaling it. There is also much arbitrariness in the choice of the finite part of the renormalization counterterms. This is also reflected in the predicted running of the coupling constant. However, when the masses can be neglected the leading order beta function is uniquely fixed and one obtains $\beta_{e} \sim$ $e^{3} / 12 \pi^{2}$ for large $\mu / m$. In general, when masses are not negligible, the beta function inherits a dependence on the chosen subtraction scheme.

Another relevant feature of renormalization is the expected decoupling of higher massive particles, as enforced by the Appelquist-Carazzone theorem [4]. This means that particles with mass higher than the relevant physical energy scale should not contribute to any computed observable. This ensures that for low energy physics we do not need to know about the related very high energy physics, hence supporting the effective field theory framework. The minimal subtraction (MS) scheme in dimensional regularization $[5,6]$ is a very efficient method used to evaluate the behavior of the running couplings. However, MS does not fulfill the decoupling theorem and one needs

\footnotetext{
*antonio.ferreiro@ific.uv.es

†navarro@ific.uv.es
}

to resort to a mass-dependent scheme to capture the low energy behavior of the beta function.

Renormalization theory has also been extended to quantized fields in curved spacetime from the early 1970 s, as reported in $[7,8]$. Here the main focus was the renormalization of the energy-momentum tensor and the evaluation of the effective action in a way consistent with general covariance. One of the major tools is the heatkernel or proper-time expansion of the Feynman propagator [9-11]. As in the case of perturbative computations in Minkowski space, quantized fields in curved space are also plagued with ultraviolet divergences. The DeWittSchwinger expansion serves to identify the emerging ultraviolet divergences, some of which are intrinsically tied to the spacetime curvature and are absent in flat space. In the evaluation of the renormalized effective action the removal of the divergences can also be done using a mass independent scheme, like MS in dimensional regularization [12]. This introduces the usual $\mu$ parameter and the associate running of the gravitational coupling constants (see, for instance, [7,13]). As expected, the obtained runnings do not fulfill the Appelquist-Carazzone theorem and in consequence make it difficult to arrive at any physical interpretation in the cosmic infrared regime. This is specially important in discussing the cosmological constant problem and the running of Newton's constant [13-15].

In this work we propose to reevaluate the effective action, and the associated beta functions, by reexpressing the conventional DeWitt-Schwinger adiabatic expansion with the introduction of a novel $\mu$ scale parameter in the definition of the adiabatic subtraction terms. The $\mu$ parameter is introduced in such a way that a natural decoupling emerges in the running couplings. We also show how the vacuum metamorphosis model $[16,17]$, one of the most 
appealing models to account for dark energy $[18,19]$ and to soften the measured $H_{0}$ tension [20], emerges when the $\mu$ parameter is interpreted in terms of the Ricci scalar.

To make the paper self-contained we first introduce the DeWitt-Schwinger (proper-time) expansion and briefly summarize the derivation of the well-known running for the couplings in dimensional regularization with the minimal prescription. To better explain the main ideas we consider a quantized complex scalar field coupled to external gravitational and electromagnetic fields. The introduction of the external electromagnetic field is somewhat tangential to the main topic of the paper. However, we introduce it in the discussion for pedagogical purposes, since the running of the effective electric charge is a wellestablished theoretical and experimental result. This permits one to compare the one-loop electromagnetic behavior with analogous results in gravity. We use units for which $c=1=\hbar$. Our sign conventions for the signature of the metric and the curvature tensor follow Refs. [7,8].

\section{EFFECTIVE ACTION, DEWITT-SCHWINGER EXPANSION, AND MINIMAL SUBTRACTION}

We start from the classical Einstein-Maxwell theory

$S=\int d^{4} x \sqrt{-g}\left(-\Lambda+\frac{R}{16 \pi G}-\frac{1}{4 q^{2}} F_{\mu \nu} F^{\mu \nu}\right)+S_{\mathrm{M}}$

coupled to a quantized charged scalar field described by the action

$S_{\mathrm{M}}=\int d^{4} x \sqrt{-g}\left(\left(D_{\mu} \phi\right)^{\dagger} D^{\mu} \phi+m^{2}|\phi|^{2}+\xi R|\phi|^{2}\right)$,

with $D_{\mu}=\nabla_{\mu}+i A_{\mu}$. The most relevant physical objects are the renormalized energy-momentum tensor $\left\langle T_{\mu \nu}\right\rangle$ and the one-loop effective action $S_{\text {eff }}$ for the matter field, related by $\frac{2}{\sqrt{-g}} \frac{\delta S_{\text {eff }}}{\delta g^{\prime \nu}}=\left\langle T_{\mu \nu}\right\rangle$. The effective action can be formally expressed in terms of the Feynman propagator $S_{\text {eff }}=$ $-i \operatorname{Tr} \log \left(-G_{F}\right)$. The propagator satisfies the KleinGordon type equation

$$
\left(\square_{x}+m^{2}+\xi R\right) G_{\mathrm{F}}\left(x, x^{\prime}\right)=-|g(x)|^{-1 / 2} \delta\left(x-x^{\prime}\right) .
$$

In general, the above formal expression for the effective action is divergent. To explicitly identify the ultraviolet divergences, one can express the Feynman propagator as an integral in the proper time $s$

$$
G_{\mathrm{F}}\left(x, x^{\prime}\right)=-i \int_{0}^{\infty} d s e^{-i m^{2} s}\left\langle x, s \mid x^{\prime}, 0\right\rangle,
$$

where $m^{2}$ is understood to have an infinitesimal negative imaginary part $\left(m^{2} \equiv m^{2}-i \epsilon\right)$. The kernel $\left\langle x, s \mid x^{\prime}, 0\right\rangle$ can be expanded in powers of the proper time as follows: $\left\langle x, s \mid x^{\prime}, 0\right\rangle=i \frac{\Delta^{1 / 2}\left(x, x^{\prime}\right)}{(4 \pi)^{2}(i s)^{2}} \exp \frac{\sigma\left(x, x^{\prime}\right)}{2 i s} \sum_{j=0}^{\infty} a_{j}\left(x, x^{\prime}\right)(i s)^{j}$

$\left[\Delta\left(x, x^{\prime}\right)\right.$ is the Van Vleck-Morette determinant and $\sigma\left(x, x^{\prime}\right)$ is the proper distance along the geodesic from $x^{\prime}$ to $x$ ]. Therefore, the effective Lagrangian, defined as $S_{\text {eff }}=$ $\int d^{4} x \sqrt{-g} L_{\text {eff }}$, has the following asymptotic expansion:

$$
L_{\mathrm{eff}}=\frac{2 i}{2(4 \pi)^{2}} \sum_{j=0}^{\infty} a_{j}(x) \int_{0}^{\infty} e^{-i s m^{2}}(i s)^{j-3} d s .
$$

The first coefficients $a_{n}\left(x, x^{\prime}\right)$ are given, in the coincidence limit $x \rightarrow x^{\prime}$, by $[7,8]$

$$
\begin{aligned}
a_{0}(x)= & 1, \quad a_{1}(x)=-\bar{\xi} R, \\
a_{2}(x)= & \frac{1}{180} R_{\alpha \beta \gamma \delta} R^{\alpha \beta \gamma \delta}-\frac{1}{180} R^{\alpha \beta} R_{\alpha \beta} \\
& -\frac{1}{6}\left(\frac{1}{5}-\xi\right) \square R+\frac{1}{2} \bar{\xi}^{2} R^{2}-\frac{1}{12} F^{\mu \nu} F_{\mu \nu},
\end{aligned}
$$

where $\bar{\xi}=\xi-\frac{1}{6}$. We remark that all dependence on the mass is factored out in the exponential in (4) [or, equivalently, in (6)]. Furthermore, all DeWitt-Schwinger coefficients $a_{n}$ are polynomial functions of the basic objects: curvature tensors $R_{\alpha \beta \gamma \delta}, F_{\mu \nu}$ (and their covariant derivatives), and the metric tensor $g^{\mu \nu}$. The removal of divergences is usually done via dimensional regularization and minimal subtraction.

In $n$ spacetime dimensions the corresponding expression (6) can be expanded as

$L_{\mathrm{eff}} \approx \frac{2 i}{2(4 \pi)^{n / 2}}\left(\frac{m}{\mu}\right)^{n-4} \sum_{j=0}^{\infty} a_{j}(x) m^{4-3 j} \Gamma\left(j-\frac{n}{2}\right)$,

where one has introduced an arbitrary mass scale $\mu$ to maintain the initial units of $L_{\text {eff }}$ as (length) $)^{4} . \mu$ is an arbitrary scale, totally independent of $m$. As $n \rightarrow 4$, the first three terms diverge with simple poles in $1 /(n-4)$. Subtracting the terms with poles one obtains an asymptotic expression for the renormalized effective Lagrangian. This also requires that the original classical Lagrangian be modified, up to total derivatives, by the addition of higher derivative terms of the form $\alpha_{1} C^{2}+\alpha_{2} R^{2}$, where $\alpha_{1}$ and $\alpha_{2}$ are dimensionless coupling constants. Here $C^{2} \equiv$ $R_{\mu \nu \alpha \beta} R^{\mu \nu \alpha \beta}-2 R_{\mu \nu} R^{\mu \nu}+\frac{1}{3} R^{2}$ is the square of the Weyl tensor. Demanding that the total effective Lagrangian, including the classical part, be $\mu$ independent leads to the following beta functions (see for instance [13]) 
$\beta_{\Lambda}^{M S}=\frac{m^{4}}{16 \pi^{2}}, \quad \beta_{\kappa}^{M S}=-\frac{m^{2} \bar{\xi}}{4 \pi^{2}}, \quad \beta_{q}^{M S}=\frac{q^{3}}{48 \pi^{2}}$,

$\beta_{\alpha_{1}}^{M S}=-\frac{1}{960 \pi^{2}}, \quad \beta_{\alpha_{2}}^{M S}=-\frac{1}{16 \pi^{2}} \bar{\xi}^{2}$,

where $\kappa^{-1}=8 \pi G$. The unsatisfactory point of the above results is the absence of decoupling for heavy massive fields.

\section{ADIABATIC DEWITT-SCHWINGER SUBTRACTIONS: MASSLESS CASE}

The DeWitt-Schwinger expansion can also be regarded as an adiabatic expansion in number of derivatives of the metric and the external fields. This is even more explicit in its counterpart expansion in local-momentum space [21]. The high frequency behavior of the Feynman propagator is captured by the DeWitt-Schwinger expansion, irrespective of the background dynamics. Therefore, the renormalization of the effective action can also be performed simply by subtracting off all (DeWitt-Schwinger) terms up and including the fourth adiabatic order [7]

$$
L_{\mathrm{div}}=\frac{2 i}{2(4 \pi)^{2}} \sum_{j=0}^{2} a_{j}(x) \int_{0}^{\infty} e^{-i s m^{2}}(i s)^{j-3} d s .
$$

However, as stressed in [22], the DeWitt-Schwinger subtractions are in general ill defined for $m=0$, due to an infrared divergence in the integration of the heat kernel. More precisely, the DeWitt-Schwinger representation of the Feynman propagator can be regarded as a special case of the Hadamard expansion, corresponding to a particular choice of the undetermined biscalar coefficient $\omega_{0}$ in the Hadamard representation [22]. The DeWitt-Schwinger expansion corresponds to the choice $\omega_{0}=\omega_{0}^{M}+\alpha a_{1}+$ $\frac{a_{2}}{m^{2}}+\frac{a_{3}}{m^{4}} \cdots \omega_{0}^{M}$ is the constant value in Minkowski space (see for instance [23]). In some special situations, as in the evaluation of trace anomalies, one can bypass this potential problem by taking the massless limit at the end of the calculation [8]. The result turns out to be finite.

Here we take a different route. When $m=0$ one can alternatively bypass this infrared issue by introducing a mass scale parameter $\mu$. It can also serve to define the necessary (but arbitrary) renormalization point. We note that this is somewhat similar to the introduction of the arbitrary length scale $\lambda \sim 1 / \mu$ in the logarithm term $V\left(x, x^{\prime}\right) \log \frac{\sigma\left(x, x^{\prime}\right)}{\lambda^{2}}$ of the Hadamard expansion [24]. For massive fields, the natural length scale is $\lambda \sim m^{-1}$. However, for massless fields one is forced to introduce the arbitrary scale $\lambda$.

In the DeWitt-Schwinger expansion one can naturally replace the mass parameter $m^{2}$ in (10) by an arbitrary $\mu^{2}$ parameter and redefine the DeWitt coefficients $a_{i} \rightarrow \bar{a}_{i}$ to keep consistency within each adiabatic order. The new proposed $L_{\text {div }}(\mu)$ reads

$$
L_{\mathrm{div}}(\mu)=\frac{2 i}{2(4 \pi)^{2}} \sum_{j=0}^{2} \bar{a}_{j}(x) \int_{0}^{\infty} e^{-i s \mu^{2}}(i s)^{j-3} d s,
$$

where the first coefficients $\bar{a}_{i}$ of the expansion are

$$
\begin{aligned}
& \bar{a}_{0}(x)=1, \quad \bar{a}_{1}(x)=a_{1}(x)+\mu^{2}, \\
& \bar{a}_{2}(x)=a_{2}(x)+\bar{\xi} R \mu^{2}+\frac{1}{2} \mu^{4} .
\end{aligned}
$$

Now we can separate from expression (11) a $\mu$-independent divergent term and a finite $\mu$-dependent term by computing the finite expression

$$
L_{\mathrm{div}}(\mu)-L_{\mathrm{div}}\left(\mu_{0}\right)=\delta_{\Lambda}+\delta_{G} R+\delta_{\sigma} a_{2}
$$

where $\quad \delta_{\Lambda}=\frac{-1}{(8 \pi)^{2}}\left(\mu^{4}-\mu_{0}^{4}\right) ; \quad \delta_{G}=\frac{1}{16 \pi^{2}} \bar{\xi}\left(\mu^{2}-\mu_{0}^{2}\right) ; \quad$ and $\delta_{\sigma}=\frac{-1}{16 \pi^{2}} \log \left(\mu^{2} / \mu_{0}^{2}\right)$. A consequence of the introduction of the arbitrary scale $\mu$ is the natural emergence of the renormalization group flow [25]. The beta functions are obtained by requiring $\mu$ independence of the effective Lagrangian

$$
\begin{aligned}
L_{\text {eff }}= & -\Lambda(\mu)+\frac{1}{2} \kappa(\mu) R-\frac{1}{4 q^{2}(\mu)} F_{\mu \nu} F^{\mu \nu} \\
& +\alpha_{1}(\mu) C^{2}+\alpha_{2}(\mu) R^{2}+\alpha_{3}(\mu) E+\alpha_{4}(\mu) \square R \\
& -\left(\delta_{\Lambda}(\mu)+\delta_{G}(\mu) R+\delta_{\sigma}(\mu) a_{2}\right)+\cdots .
\end{aligned}
$$

$E=R_{\mu \nu \alpha \beta} R^{\mu \nu \alpha \beta}-4 R_{\mu \nu} R^{\mu \nu}+R^{2}$ is the integrand of the Gauss-Bonet topological invariant. Note that the omitted terms in the third line of (14) are independent of $\mu$. The results for the beta functions are

$$
\begin{array}{ll}
\beta_{\Lambda}=\frac{\mu^{4}}{16 \pi^{2}}, & \beta_{\kappa}=\frac{\bar{\xi} \mu^{2}}{4 \pi^{2}}, \\
\beta_{q}=\frac{q^{3}}{48 \pi^{2}}, & \beta_{1}=\frac{-1}{960 \pi^{2}}, \quad \beta_{2}=\frac{-\bar{\xi}^{2}}{16 \pi^{2}}, \\
\beta_{3}=\frac{1}{2880 \pi^{2}}, & \beta_{4}=\frac{\frac{1}{5}-\xi}{48 \pi^{2}} .
\end{array}
$$

We have included for completeness all coupling constants. This agrees with the results obtained in [26] for Friedmann-Lemaitre-Robertson-Walker spacetimes using a similar generalization of the usual adiabatic regularization method [27], via the introduction of an analogous off-shell scale $\mu$. (For a recent use of this generalization see [28]). We also have exact agreement for the dimensionless coupling constants obtained from MS, as displayed in (9). Hadamard renormalization also leads to a similar result for the running of the electric coupling constant $[24,29]$. 


\section{MASSIVE CASE, DECOUPLING AND RUNNING GRAVITATIONAL CONSTANTS}

Now we want to generalize the previous analysis to massive fields. Therefore, instead of (11) and (10) we should write

$L_{\mathrm{div}}(\mu)=\frac{2 i}{2(4 \pi)^{2}} \sum_{j=0}^{2} \bar{a}_{j}(x) \int_{0}^{\infty} e^{-i s f\left(\mu^{2}, m^{2}\right)}(i s)^{j-3} d s$.

The simplest choice for the function $f\left(\mu^{2}, m^{2}\right)$ is $f\left(\mu^{2}, m^{2}\right)=m^{2}+\mu^{2}$. This choice is univocally singularized if we demand that the mass $m^{2}$ is factored out in the form of an exponential $e^{-i s m^{2}}$, as in the conventional DeWitt-Schwinger expansion (10). Furthermore, for $m=$ 0 we have to recover (11). Hence

$L_{\mathrm{div}}(\mu)=\frac{2 i}{2(4 \pi)^{2}} \sum_{j=0}^{2} \bar{a}_{j}(x) \int_{0}^{\infty} e^{-i s\left(m^{2}+\mu^{2}\right)}(i s)^{j-3} d s$,

$\bar{a}_{0}(x)=1, \quad \bar{a}_{1}(x)=a_{1}(x)+\mu^{2}, \quad$ and $\quad \bar{a}_{2}(x)=a_{2}(x)+$ $\bar{\xi} R \mu^{2}+\frac{1}{2} \mu^{4}$ are kept mass independent. Note that any other expression for $f$ implies dependence on the mass of the redefined DeWitt coefficients $\bar{a}_{i}$. Even more, any other choice for $f$ implies a nonpolynomial dependence of the coefficients $\bar{a}_{i}$ on $m^{2}$.

The corresponding beta function for the electric charge obtained from (17) is

$$
\beta_{q}=\frac{q^{3}}{48 \pi^{2}} \frac{\mu^{2}}{m^{2}+\mu^{2}},
$$

while the result for the dimensionless gravitational constants are similarly

$$
\begin{array}{ll}
\beta_{1}=-\frac{1}{960 \pi^{2}} \frac{\mu^{2}}{m^{2}+\mu^{2}}, & \beta_{2}=-\frac{\xi^{2}}{16 \pi^{2}} \frac{\mu^{2}}{m^{2}+\mu^{2}}, \\
\beta_{3}=\frac{1}{2880 \pi^{2}} \frac{\mu^{2}}{m^{2}+\mu^{2}}, & \beta_{4}=\frac{\frac{1}{5}-\xi}{48 \pi^{2}} \frac{\mu^{2}}{m^{2}+\mu^{2}} .
\end{array}
$$

The difference between (18)-(19) and (15) is that the former approaches the latter in the limit $\mu \gg m$ while it approaches to zero quadratically in the limit $\mu \ll m$. This is equivalent to the decoupling of very massive charged particles in scalar electrodynamics.

Concerning the dimensionfull gravitational constants, the decoupling is also absent in dimensional regularization. This makes it not trivial to assign some physical meaning to the $\mu$ parameter. However, within the proposed DeWittSchwinger framework and from (17) we get the following beta functions:

$$
\beta_{\Lambda}=\frac{1}{16 \pi^{2}} \frac{\mu^{6}}{m^{2}+\mu^{2}}, \quad \beta_{\kappa}=\frac{\bar{\xi}}{4 \pi^{2}} \frac{\mu^{4}}{m^{2}+\mu^{2}} .
$$

For large values of the scale $\mu \gg m$ the mass can be ignored, while heavy particles $m \gg \mu$ decouple and the beta functions tend to zero. Note that the decoupling of the dimensionfull gravitational constants, in contrast with the dimensionless ones, is a highly nontrivial issue [30-34].

The running of the cosmological and Newton's gravitational constants are given by $\left(\Lambda=\Lambda_{c} / 8 \pi G\right.$, where $\Lambda_{c}$ is the traditional cosmological constant)

$$
\begin{aligned}
\Lambda(\mu)= & \Lambda_{0}+\frac{1}{64 \pi^{2}}\left(\left(\mu^{4}-\mu_{0}^{4}\right)-2 m^{2}\left(\mu^{2}-\mu_{0}^{2}\right)\right. \\
& \left.+2 m^{4} \log \left(\frac{m^{2}+\mu^{2}}{m^{2}+\mu_{0}^{2}}\right)\right) \\
& G(\mu)=\frac{G_{0}}{1+\frac{\bar{\xi} G_{0}}{\pi}\left(\mu^{2}-\mu_{0}^{2}-m^{2} \log \left(\frac{m^{2}+\mu^{2}}{m^{2}+\mu_{0}^{2}}\right)\right)},
\end{aligned}
$$

while the running for the dimensionless gravitational constants are

$$
\alpha_{i}(\mu)=\alpha_{i 0}+\frac{\sigma_{i}}{4 \pi^{2}} \log \left(\frac{m^{2}+\mu^{2}}{m^{2}+\mu_{0}^{2}}\right)
$$

where $\sigma_{1}=-\frac{1}{430}, \sigma_{2}=-\frac{\bar{\xi}^{2}}{8}, \sigma_{3}=\frac{1}{1440}$, and $\sigma_{4}=\frac{(1 / 5-\xi)}{24}$.

\section{RELATION WITH OTHER APPROACHES}

It is interesting to briefly consider the massless limit for the predicted running for the Newton constant, as given by (22): $G(\mu)=G_{0}\left(1+(\bar{\xi} / \pi) G_{0}\left(\mu^{2}-\mu_{0}^{2}\right)\right)^{-1}$. This expression has the same form as the one obtained within a very different approach. The asymptotic safety framework of quantum gravity predicts a similar behavior for the running of Newton's constant [35] (see also [36]).

Even though the above renormalization prescription does not give us a uniquely physical interpretation for $\mu$, it supports the idea that indeed it can be linked to some physical scale, such as the conventional momentum scaling $p_{i} \rightarrow s p_{i}$ in flat space particle scattering associated with the scaling $\mu \rightarrow s \mu_{0}$. In curved spacetime the scaling of $\mu$ should be linked, by dimensional reasons, to the scaling of the metric $g_{\mu \nu} \rightarrow s^{-2} g_{\mu \nu}$, and hence to the scaling of the curvature $R \rightarrow s^{2} R$ [37]. Therefore, while the dependence on $\mu$ of the renormalized electric charge has the same form as the dependence of the measured charge on the square of the momentum transfer in electron scattering, the dependence of the renormalized $\Lambda$ or $\kappa$ on $\mu$ is expected to be traded to the curvature dependence of the observable gravitational constants. One possible way of choosing a natural mass/length scale in a cosmological setting is to make $\mu$ proportional to the Hubble parameter $H$, or $\mu^{2}$ to be 
proportional to the Ricci scalar $R$. Here we are more interested in the infrared behavior of the runnings, and hence in the low curvature regime. The runnings obtained above are somewhat similar to the generic form of the running proposed in the running vacuum models [38] (see also $[13,28,39]$ for a connection with cosmological observations and smoothing of data tensions). The connection between $\mu^{2}$ and $R$ has also been previously suggested in [31].

Let us analyze with more details the consequences of the assumption $\mu^{2} \propto R$. For computational purposes it is convenient to choose $\mu^{2}=\bar{\xi} R$. We also select the reference point $\mu_{0}=0$ and assume that

$$
\Lambda_{0}=0, \quad \alpha_{i 0}=0,
$$

and keep $\kappa_{0}=\left(1 / 8 \pi G_{0}\right)$, where $G_{0}$ is the measured Newton's constant. These renormalization conditions can be understood as our definition of the physical gravitational constants in the very infrared limit point. The effective Lagrangian is well approximated, in the adiabatic limit of our late-time expanding Universe, by (here we are considering a single real scalar field)

$$
\begin{aligned}
L_{\text {eff }}= & -\Lambda(\mu)+\frac{1}{2} \kappa(\mu) R+\alpha_{1}(\mu) C^{2}+\alpha_{2}(\mu) R^{2} \\
& ++\alpha_{3}(\mu) E+\alpha_{4}(\mu) \square R .
\end{aligned}
$$

Taking into account the running derived in (21)-(23) for all gravitational coupling constants and the conditions (24), the above effective action can be rewritten in the form

$$
\begin{aligned}
L_{\text {eff }}= & \frac{1}{2} \kappa_{0} R+\frac{1}{64 \pi^{2}}\left\{m^{2} \bar{\xi} R+\frac{3}{2} \bar{\xi}^{2} R^{2}\right. \\
& \left.-\left(m^{4}+2 m^{2} \bar{\xi} R+2 a_{2}\right) \log \left(\frac{m^{2}+\bar{\xi} R}{m^{2}}\right)\right\} .
\end{aligned}
$$

Remarkably, this coincides with the action proposed by Parker and Raval in [16,17], and known as the vacuum metamorphosis model $[18,19]$, on the basis of the $R$-summed form of the Feynman propagator [40-42]. Here only the measured Newton's constant $G_{0}$ appears in the action. The semiclassical dynamics of (26) provides negative pressure to suddenly accelerate the Universe at a rate compatible with observations (it softens also the $H_{0}$ tension [20]) for an ultralow mass scalar field, of the same order as the current expansion rate of the Universe $\left(m \sim H_{0}\right)$, in accordance with the underlying decoupling mechanism displayed above. This provides further evidence for the connection between the parameter $\mu$ in our proposed physical renormalization scheme with the physical scale $R$.

\section{CONCLUSIONS AND FINAL COMMENTS}

We have generalized the DeWitt-Schwinger renormalization subtractions to include an arbitrary renormalization mass scale $\mu$, and in such a way to ensure the decoupling of heavy masses. This is a somewhat improvement with respect to the more common treatment of the DeWittSchwinger expansion via dimensional regularization and minimal subtraction. We have also analyzed the new predicted running for the gravitational couplings.

As a by-product of our proposal, and because of the natural decoupling, the obtained runnings could be of interest for the issue of the cosmological constant problem. To see this in the conventional way let us assume that $\Lambda_{0}=0$. Following the standard approach, i.e., dimensional regularization and MS, any massive particle will contribute as $\Lambda^{\mathrm{MS}}(\mu) \sim m^{4} \log \left(\frac{\mu^{2}}{m^{2}}\right)$ (see, for instance, [13,14]) and taking the characteristic scale of the Standard Model gives the well-known extremely high contribution $\Lambda \sim 10^{46} \mathrm{eV}^{4}$. This is in conflict with the observed current energy density $\Lambda_{\text {obs }} \sim 10^{-11} \mathrm{eV}^{4}$ (see [13] for a detailed discussion). However, if we now use (21) we obtain an extremely low value. More generally, in the limit of large masses $m \gg$ $\mu \sim H_{0}$ (all the standard model particles) the term $\mathrm{m}^{4}$ decouples and we get $\Lambda^{D S}(\mu) \sim \frac{\mu^{6}}{m^{2}}+\mathcal{O}\left(\frac{1}{m^{4}}\right)$. This heuristic discussion suggests that the origin of the accelerated expansion could be more naturally found in ultralow masses. This requires the identification of $\mu^{2}$ as a timedependent scale proportional to the Ricci scalar, as also reinforced in the more quantitative arguments displayed in this work. Further work is required to make more definite statements.

\section{ACKNOWLEDGMENTS}

We thank P. Beltran-Palau, S. A. Franchino-Vinas, S. Nadal, and S. Pla for useful comments. This work has been supported by the Spanish MINECO research Grants No. FIS2017-84440-C2-1-P and No. FIS2017-91161EXP. A.F. is supported by the Severo Ochoa Ph.D. fellowship, Grant No. SEV-2014-0398-16-1, and the European Social Fund. 
[1] S. Weinberg, The Quantum Theory of Fields (Cambridge University Press, Cambridge, England, 1995), Vol. 1-2.

[2] M. E. Peskin and D. V. Schroeder, An Introduction to Quantum Field Theory (Addison-Wesley, Reading, MA, 1995).

[3] L. Alvarez-Gaume and M. A. Vazquez-Mozo, An Invitation to Quantum Field Theory (Springer-Verlag, Berlin, 2012).

[4] T. Appelquist and J. Carazzone, Phys. Rev. D 11, 2856 (1975).

[5] G. 't Hooft, Nucl. Phys. B61, 455 (1973).

[6] G. 't Hooft and M. J. G. Veltman, Nucl. Phys. B44, 189 (1972).

[7] L. Parker and D. J. Toms, Quantum Field Theory in Curved Spacetime: Quantized Fields and Gravity (Cambridge University Press, Cambridge, England, 2009).

[8] N. D. Birrell and P. C. W. Davies, Quantum Fields in Curved Space (Cambridge University Press, Cambridge, England, 1982).

[9] J. Schwinger, Phys. Rev. 82, 664 (1951).

[10] B.S. DeWitt, Dynamical Theory of Groups and Fields (Gordon and Breach, New York, 1965).

[11] B. S. DeWitt, Phys. Rep. 19, 295 (1975).

[12] T. S. Bunch, J. Phys. A 12, 517 (1979).

[13] J. Solà, J. Phys. Conf. Ser. 453, 012015 (2013).

[14] J. Martin, C. R. Phys. 13, 566 (2012).

[15] S. M. Carroll, Living Rev. Relativity 4, 1 (2001).

[16] L. Parker and A. Raval, Phys. Rev. D 60, 063512 (1999).

[17] L. Parker and A. Raval, Phys. Rev. D 62, 083503 (2000); Phys. Rev. Lett. 86, 749 (2001).

[18] L. Parker and D. A. T. Vanzella, Phys. Rev. D 69, 104009 (2004).

[19] R. R. Caldwell, W. Komp, L. Parker, and D. A. T. Wanzella, Phys. Rev. D 73, 023513 (2006).

[20] E. Di Valentino, E. V. Linder, and A. Melchiorri, Phys. Rev. D 97, 043528 (2018).

[21] T. S. Bunch and L. Parker, Phys. Rev. D 20, 2499 (1979).

[22] R. M. Wald, Quantum Field Theory in Curved Spacetime and Black Hole Thermodynamics (University of Chicago Press, Chicago, 1994).

[23] M. A. Castagnino and D. D. Harari, Ann. Phys. (N.Y.) 152, 85 (1984).
[24] V. Balakumar and E. Winstanley, Classical Quantum Gravity 37, 065004 (2020).

[25] S. Coleman, Aspects of Symmetry (Cambridge University Press, Cambridge, England, 1985).

[26] A. Ferreiro and J. Navarro-Salas, Phys. Lett. B 792, 81 (2019).

[27] L. Parker and S. A. Fulling, Phys. Rev. D 9, 341 (1974); P. R. Anderson and L. Parker, Phys. Rev. D 36, 2963 (1987); I. Agullo, J. Navarro-Salas, G. J. Olmo, and L. Parker, Phys. Rev. D 84, 107304 (2011).

[28] C. Moreno-Pulido and J. Sola, Eur. Phys. J. C 80, 692 (2020).

[29] P. Beltran-Palau, J. Navarro-Salas, and S. Pla, Phys. Rev. D 101, 105014 (2020).

[30] E. V. Gorbar and I. L. Shapiro, J. High Energy Phys. 02 (2003) 021.

[31] T. Markkanen, Phys. Rev. D 91, 124011 (2015).

[32] A. Babic, B. Guberina, R. Horvat, and H. Stefancic, Phys. Rev. D 65, 085002 (2002); 71, 124041 (2005).

[33] S. A. Franchino-Vinas, T. D. Netto, I. L. Shapiro, and O. Zanusso, Phys. Lett. B 790, 229 (2019).

[34] S. A. Franchino-Vinas, T. de Paula Netto, and O. Zanusso, Universe 5, 67 (2019).

[35] M. Niedermaier and M. Reuter, Living Rev. Relativity 9, 5 (2006); M. Reuter, arXiv:hep-th/0012069.

[36] A. Polyakov, in Gravitation and Quantization, edited by J. Zinn-Justin and B. Julia (North-Holland, Amsterdam, 1995).

[37] B. Nelson and P. Panangaden, Phys. Rev. D 25, 1019 (1982); L. Parker and D. Toms, Phys. Rev. D 29, 1584 (1984); S. Hollands and R. M. Wald, Commun. Math. Phys. 237, 123 (2003).

[38] I. L. Shapiro and J. Solà, J. High Energy Phys. 02 (2002) 006; Phys. Lett. B 475, 236 (2000); 682, 105 (2009).

[39] J. Solà, A. Gómez-Valent, and J. de Cruz Pérez, Astrophys J. 836, 43 (2017); J. Solà, Int. J. Mod. Phys. A 33, 1844009 (2018).

[40] L. Parker and D. J. Toms, Phys. Rev. D 31, 953 (1985).

[41] I. Jack and L. Parker, Phys. Rev. D 31, 2439 (1985).

[42] A. Ferreiro, J. Navarro-Salas, and S. Pla, Phys. Rev. D 101, 105011 (2020). 